\title{
MOEA: formando líderes para las Américas
}

\author{
Kenyi Díaz Oshiyama y Renzo Miranda León
}

\section{Agradecimiento}

Antes de iniciar la redacción del presente artículo, es justo y necesario dedicar este apartado a agradecer a todas aquellas personas que hicieron posible que el Modelo de la Asamblea General de la OEA para Universidades del Hemisferio (MOEA), que parecía un sueño, se vuelva realidad. En primer lugar, gracias a las autoridades de la Universidad de Lima, a la Facultad de Derecho y al doctor Oswaldo Hundskopf, su decano, por todo el apoyo brindado. Por supuesto, infinitas gracias a la profesora Carolina Loayza por apostar, y seguir apostando, por el desarrollo del MOEA en la Universidad de Lima, lo cual permitió que nos animáramos a participar del Taller de Selección y asumiéramos el reto de postular para ser parte de la delegación. Muchas gracias por sus conocimientos, su dedicación y sus consejos.

Asimismo, un agradecimiento muy especial a los diplomáticos peruanos que colaboraron en nuestra preparación. Gracias también a la Representación Permanente del Perú ante la OEA, a la Oficina de la OEA en Perú; $y$, por supuesto, a los organizadores del XXXI MOEA en Washington D.C., y del XXXII MOEA en Arequipa.

Una mención especial merecen nuestros compañeros de delegación: María Teresa Novoa, David Baracco, Susana Ramos, Francisco Escudero, 
Paul Escobar, Silvia Guevara, Keyla Bautista, Carlos Fernández y, nuestra comunicadora, Karla Renjifo, quienes participaron en el XXXI MOEA; así como los miembros de la delegación de la Universidad de Lima que participaron en el XXXII MOEA: Giuliana Temoche, Pierina Gonzales, Miluzka Contreras, Ingrid Jáuregui, Susana Santos, Percy Torrelio, Mariana Lazo, María Ximena Zegarra, Estela Anaya y Diego Rodríguez. Grandes personas, grandes líderes.

Finalmente, un agradecimiento muy especial a Ius et Praxis, revista oficial de la Facultad de Derecho de la Universidad de Lima, por brindarnos el espacio para difundir este tipo de prácticas académicas de carácter internacional, que resultan muy beneficiosas para nuestro desarrollo personal como estudiantes y para continuar trabajando por el desarrollo de nuestro continente.

\section{Introducción}

Al hablar sobre actividades académicas internacionales, es inevitable tocar el tema de la globalización y de la sociedad del conocimiento en la cual vivimos desde hace un poco más de dos décadas. Estos dos factores han hecho que nuestras vidas cambien por completo y que ahora podamos realizar actividades que antes eran inimaginables. En consecuencia, podemos afirmar que, por ejemplo, las exigencias académicas para postular a una beca o a un trabajo cada vez son más elevadas. Es decir, no solo un estudiante o profesional es competente por poseer conocimientos, saber inglés y programas informáticos, además debe contar con ciertas habilidades y destrezas psicosociales, como el liderazgo, todo lo cual le permitirá poseer un perfil competitivo a nivel internacional.

Pues bien, es de esta manera como los responsables de seleccionar a los estudiantes y profesionales que postulan a una pasantía en el Banco Interamericano de Desarrollo (BID) nos lo explicaron, en una reunión convocada por la Representación Permanente del Perú ante la OEA y a la cual asistieron todas las delegaciones peruanas que participaron en el XXXI MOEA. En ese sentido, es de suma importancia aprovechar todas las oportunidades académicas internacionales que se presenten, con el fin de poder desarrollar las mencionadas destrezas y habilidades. En este punto, nos estamos refiriendo a la participación en Modelos de Asambleas Generales de Organizaciones Internacionales, como los Modelos de las Naciones Unidas (MUN) o el MOEA; así como a la postulación a 
pasantías en organismos internacionales, como la OEA ${ }^{1}$, el BID, la Corte Interamericana de Derechos Humanos, el Banco Mundial, entre otros.

Sin embargo, la oportunidad de haber participado en el MOEA nos ha permitido darnos cuenta de que este Modelo brinda un plus: tiene como objetivo desarrollar en la juventud americana los valores democráticos que faciliten la permanencia de la democracia en nuestro continente, de manera que, de acuerdo a la Carta Democrática Interamericana (Organización de los Estados Americanos, s.f.), se llegue a alcanzar el desarrollo integral de los pueblos americanos. Todo ello sin perder de vista el impacto que tiene el Modelo en la vida personal de cada estudiante participante, como el desarrollo de una conciencia crítica frente a los problemas interamericanos y la defensa de los intereses nacionales.

Siendo así, es importante tener presente que, a nosotros, estudiantes de derecho, nos forman para confrontar, es decir, para defender una postura a través del debate. La mayoría de las veces ya no pensamos en la conciliación para poder solucionar una controversia, a pesar de que los medios de solución autocompositivos han permitido solucionar controversias fundamentales para la historia de la humanidad. De acuerdo a ello, podemos afirmar, como un adelanto, que el MOEA nos ayudó a plantear importantes alternativas de solución a los problemas de las Américas (incluidos en el temario), a través del consenso y de las prácticas diplomáticas.

Ahora bien, el MOEA (o MOAS, en inglés: Model of the Organization of American States) es el Modelo de la Asamblea General de la Organización de los Estados Americanos para Universidades del Hemisferio. De acuerdo a la página web del Modelo, "el MOEA es el ejercicio de simulación en el cual los estudiantes representan a los diplomáticos de los Estados miembros de la OEA y debaten resoluciones sobre los temas actuales de la Agenda Interamericana" (OEA, s.f.).

En ese orden de ideas, es importante señalar que la Universidad de Lima viene participando en el MOEA desde 2010, de manera ininterrumpida:

- $\quad$ En 2010, participó representando a Colombia en el MOEA realizado en Lima, Perú.

1 Para mayor información: Organización de los Estados Americanos. "Programa de Pasantías de la OEA" [en línea]. <http://www.oas.org/es/saf/drh/pasantias/ indexES.asp $>$. [Consulta: 20 de enero de 2014]. 
- En 2011, participó representando a Estados Unidos en el MOEA realizado en San Salvador, El Salvador.

- En 2012, participó representando a Guatemala en el MOEA realizado en Cochabamba, Bolivia.

- En 2013, participó representando a Jamaica en el MOEA realizado en Washington D.C., Estados Unidos.

- En 2014, participó representando a Bolivia en el MOEA realizado en Arequipa, Perú.

A continuación, detallaremos ciertos aspectos importantes del MOEA y de la exitosa participación que tuvo la delegación de la Universidad de Lima en el XXXI MOEA y en el XXXII MOEA.

\section{MOEA: datos generales y su importancia}

Tal como lo afirma la página web del MOEA, "el MOEA es un programa de la Organización de los Estados Americanos (OEA) creado con el objeto de promover los valores democráticos entre la juventud del Hemisferio, mediante un ejercicio de simulación de la Asamblea General de la OEA" (OEA, s.f.).

Este Modelo comenzó como una iniciativa de un profesor de la Universidad de Georgetown, quien, en 1980, acudió al despacho del entonces secretario general de la OEA, el embajador Alejandro Orfila, a efectos de proponerle realizar la simulación de la Asamblea General de la OEA. ¿Cuál fue la razón? Este profesor se encontraba poco conforme con la falta de conocimiento de los estudiantes acerca del sistema interamericano, a pesar de que las instalaciones de la universidad se encontraban a pocas millas de los edificios principales de la OEA (OEA, s.f.).

MOEA en español, MOAS en inglés. Es un programa diseñado para la promoción de los valores democráticos entre los estudiantes del Hemisferio. Los estudiantes que participan realizan un ejercicio de simulación, en el cual cada uno representa las políticas y los intereses de cada uno de los 34 Estados miembros de la OEA. (González, 2012)

Para ello, es importante que los estudiantes hayan investigado sobre la OEA y sus cuerpos políticos, el modus operandi (normas de procedimiento, o también denominadas normas parlamentarias); y todos los datos importantes de los estados que son parte de la OEA, como su his- 
toria, geografía, economía, estructura política, Constitución, leyes, políticas públicas, política exterior, cultura (música, gastronomía, costumbres, tradiciones y turismo), entre otros temas de importancia; de manera que ello les permita simular a diplomáticos de los Estados miembros de la OEA y defiendan los intereses de su nación. En este punto vale mencionar lo que la profesora Carolina Loayza siempre nos recalcaba: "Los Estados no tienen amigos, sino que poseen intereses, y sus representantes (gobernantes, diplomáticos y diplomáticas) deben defenderlos".

Asimismo, los estudiantes participantes deben estudiar los ítems que el temario contiene, la postura del país asignado respecto a esos temas y las resoluciones de la OEA que se relacionan con dichos temas. Para poder alcanzar estos propósitos, es importante organizar reuniones con diplomáticos de los estados que fueron asignados, así como con representantes de la OEA que se encuentran en los 34 Estados miembros.

Por otro lado, con relación a la importancia del MOEA, podemos afirmar que aquella radica en sus tres objetivos principales (OEA, s.f.):

a. Promover los valores democráticos entre la juventud del Hemisferio.

b. Familiarizar a los estudiantes, profesores e instituciones académicas con la OEA, considerando sus pilares, propósitos y principios.

c. Promover el desarrollo del liderazgo entre los jóvenes, así como habilidades de negociación, trabajo en equipo y resolución de conflictos.

Según ello, para la OEA es fundamental la participación de jóvenes en este tipo de simulaciones, pues considera que nosotros analizamos los problemas de la Agenda Interamericana desde distintas perspectivas, y, por tanto, planteamos soluciones creativas. Por esa razón, los libros de resoluciones de cada uno de los modelos son enviados al secretario general y al presidente del Consejo Permanente, con el fin de encontrar alguna idea innovadora que pueda ser incluida en una resolución real de la OEA.

En suma, la trascendencia del MOEA en la formación de un estudiante se basa en la importancia del diálogo; la formación de consenso; el trato diplomático de respeto y cordialidad; la aplicación de la negociación como herramienta para alcanzar el mencionado consenso; la buena redacción para liderar un grupo de trabajo; y la tolerancia, la empatía, la asertividad, el trabajo en equipo y el liderazgo como habilidades para interrelacionarse con los demás. 


\section{Participación de la Universidad de Lima en el XXXI MOEA}

Después de su participación en el XXX MOEA, en Cochabamba, Bolivia, la Universidad de Lima tenía que volver a prepararse para presentar una delegación para el XXXI MOEA. Es así que se organizó el Taller de Selección MOEA UL 2013, con el fin de seleccionar a los estudiantes de derecho que conformarían la delegación de la Universidad de Lima para el MOEA.

Durante las clases del Taller, aprendimos muchos temas relevantes para nuestro mejor desempeño como futuros delegados en el Modelo, tales como: la OEA, sus antecedentes históricos, misión, visión y objetivos; sus principios y pilares; sus acuerdos y tratados; sus órganos: Asamblea General, Consejo Permanente y comisiones; sus miembros y observadores; el libreto de participación y el modus operandi; la democracia, los derechos humanos, el desarrollo integral, la seguridad hemisférica, la sociedad civil, y las cuestiones administrativas y presupuestarias, y su tratamiento normativo en cada una de las comisiones del Consejo Permanente; la redacción de resoluciones; política internacional y relaciones internacionales; negociación; y etiqueta social y protocolo.

De todo ello, para nosotros, era fundamental saber que (OEA, s.f.):

a. La OEA es el organismo regional más antiguo del mundo, pues tiene sus orígenes en el Congreso Anfictiónico de Panamá de 1826, que fue convocado por el libertador Simón Bolívar.

b. Los principales pilares de la OEA son la democracia, los derechos humanos, la seguridad y el desarrollo.

c. La OEA promueve la democracia, defiende los derechos humanos, garantiza un enfoque multidimensional a la seguridad, fomenta el desarrollo integral y la prosperidad, y apoya la cooperación jurídica interamericana.

d. La OEA cuenta con una serie de órganos, entre los que se encuentran la Asamblea General y el Consejo Permanente. La Asamblea General "es el órgano supremo de la Organización de los Estados Americanos, y está compuesta por las delegaciones de todos los Estados miembros, quienes tienen derecho a hacerse representar y a emitir su voto".

Por su parte, de acuerdo al artículo $80^{\circ}$ de la Carta de la OEA, "el Consejo Permanente de la Organización se compone de un representante por cada Estado miembro, nombrado especialmente por el Gobierno respectivo con la categoría de embajador". 
A su vez, el Consejo Permanente está conformado por cinco comisiones (OEA, s.f.):

- La Comisión General, que se encarga de los siguientes temas: asistencia humanitaria y desastres; comercio; cooperación interregional; democracia; derechos humanos; niños, niñas y adolescentes en el Hemisferio; asuntos referidos a la mujer; protección del consumidor; tecnologías de la información; responsabilidad social de las empresas; entre otros.

- La Primera Comisión o Comisión de Asuntos Jurídicos y Políticos, que se encarga de los siguientes temas: derecho internacional, derechos humanos, fortalecimiento de la democracia, seguimiento de programas, entre otros.

- La Segunda Comisión o Comisión de Seguridad Hemisférica, que se encarga de los siguientes temas: acción contra las minas antipersonales; combate a la trata de personas; combate a la delincuencia organizada transnacional; combate al tráfico ilícito de armas; fomento de la confianza y de la seguridad; no proliferación de armas nucleares y educación para el desarme; prevención de la delincuencia y la violencia; reducción de desastres naturales; tratamiento de las pandillas delictivas; entre otros.

- La Tercera Comisión o Comisión sobre Gestión de Cumbres Interamericanas y Participación de la Sociedad Civil en las Actividades de la OEA, que se encarga de ver los temas que incluyen su nombre.

- La Cuarta Comisión o Comisión de Asuntos Administrativos y Presupuestarios, que se encarga de los siguientes temas: administración de los bienes inmuebles que posee la OEA; personal; cuotas de los Estados miembros; fondos y subfondos; proceso de estimación de costos de resoluciones; presupuestos; entre otros.

Luego de haber pasado por las evaluaciones correspondientes, se eligió a la delegación de la Universidad de Lima, integrada por diez estudiantes de la Facultad de Derecho y una estudiante de la Facultad de Comunicación.

A continuación, se inició el Taller de Preparación para Delegados, en el cual, además de conocer a fondo Jamaica, país que nos asignaron, su postura respecto a cada punto del temario y su relación con los demás Estados miembros de la OEA, entendimos que nuestro objetivo era utili- 
zar nuestras habilidades de negociación y consenso para lograr convencer a los delegados de cada comisión que nuestra propuesta en los grupos de trabajo era la que traía mejores beneficios para las Américas y reunía los mejores propósitos de las demás delegaciones.

Es así que, antes de partir hacia el MOEA, junto con nuestra asesora, la profesora Carolina Loayza, coordinadora del Área Internacional de la Facultad de Derecho de la Universidad de Lima, nos planteamos tres objetivos:

1. Lograr que por lo menos uno de los integrantes de la delegación pueda ser elegido como autoridad en alguna de las comisiones que conforman el Consejo Permanente de la OEA en la simulación.

2. Alcanzar que las perspectivas planteadas por la delegación sean consideradas dentro del temario de cada comisión, de manera que sirvan de referencia para la redacción de las resoluciones.

3. Conseguir que las resoluciones presentadas por los grupos de trabajo que integran los miembros de la delegación sean aprobadas en cada una de las comisiones que conforman el Consejo Permanente de la OEA en el Modelo.

Inaugurado el XXXI MOEA, el proceso de negociación y de formación del consenso se dio de la siguiente manera:

a. Los trabajos en las comisiones se iniciaron con los discursos generales de cada delegado, que versaban sobre las perspectivas que cada Estado proponía, de acuerdo al temario del Modelo.

b. Luego, continuaron las discusiones para determinar las diferentes perspectivas de cada tema, para la posterior formación de los grupos de trabajo y la emisión de un proyecto de resolución por cada grupo.

c. Una vez formados los grupos de trabajo, los delegados integrantes presentaron la postura del país asignado respecto a la perspectiva que se elaboró a partir de las discusiones iniciales, a efectos de buscar consenso sobre los puntos de las partes considerativa y resolutiva del proyecto de resolución.

d. A continuación, una vez elaborados los proyectos de resoluciones, fueron presentados por un representante del grupo de trabajo ante los delegados de cada comisión. 
e. Finalmente, se debatieron los proyectos de resolución presentados y se sometieron a votación para su aprobación en cada una de las comisiones.

Asimismo, vale indicar que, luego de todo el proceso de aprobación de las resoluciones, en cada una de las comisiones se convocó a elecciones, con el fin de elegir a las autoridades para el MOEA del siguiente año. Se eligió al presidente y al secretario general del Modelo; en la Comisión General, se eligió al vicepresidente; y en las demás comisiones, al presidente y al vicepresidente de cada comisión. En esta oportunidad, gracias al trabajo en equipo de toda la delegación, logramos obtener una autoridad: Kenyi Díaz Oshiyama fue elegido como vicepresidente de la Comisión General para el XXXII MOEA.

Es así que, después de conocer a delegados de distintos países de América $^{2}$ y de entablar con ellos intensos debates, el 26 de julio de 2013, saliendo de la sesión de clausura, pudimos decir... ¡reto cumplido!

\section{Participación de la Universidad de Lima en el XXXII MOEA}

En julio de 2014, la Universidad de Lima volvió a participar en un MOEA, esta vez en su edición XXXII, organizada en Arequipa por la Universidad Católica San Pablo. En esta oportunidad, al igual que el año anterior, se presentó una delegación conformada por diez estudiantes de la Facultad de Derecho y un estudiante de la Facultad de Comunicación, quien desempeñó el rol de oficial de información pública.

Siguiendo con las pautas establecidas por la asesora, la profesora Carolina Loayza, la delegación se planteó los objetivos a seguir, dentro de los cuales se encontraba la obtención de por lo menos dos autoridades en el Modelo. Pues bien, los objetivos se cumplieron con creces, debido a que, efectivamente, se obtuvieron las dos autoridades, siendo una de ellas la presidencia del siguiente MOEA. De esta manera, Giuliana Temoche fue elegida presidenta del Modelo e Ingrid Jáuregui presidenta de la Segunda Comisión, Comisión de Seguridad Hemisférica.

2 Asistieron delegados de Costa Rica, El Salvador, Nicaragua, Colombia, Ecuador, Venezuela, Panamá, Bolivia, Francia, Chile, Canadá, Honduras, Guyana y, por supuesto, Perú. 


\section{Temarios y su trascendencia en las Américas}

\subsection{MOEA}

En esta edición, el Modelo se desarrolló bajo un marco teórico establecido según adecuados criterios para el cumplimiento de sus objetivos. Así, el temario del Modelo, instrumento de pauta para su desenvolvimiento teórico, presentó perspectivas íntegras de los asuntos políticos más importantes de nuestra región.

\subsubsection{Declaración de Washington D.C.: "Estrategias para minimizar la} inequidad en los Estados miembros, mediante la integración económica regional de las Américas"

La Declaración del Modelo recordó la trascendencia del compromiso asumido por los Estados miembros en la Carta de la Organización de los Estados Americanos, la cual reconoce que uno de los propósitos de la Organización es promover el desarrollo económico, social, cultural y tecnológico, a través de la acción cooperativa y la observancia de principios rectores como la solidaridad y la equidad. Así, atendiendo a las actuales circunstancias del Hemisferio, la Declaración expresó no solo la necesidad de instituciones que permitan la eliminación de barreras de comercio entre los mercados de la región, sino que, al amparo de la Carta Social de las Américas, consideró imprescindible el respeto de los valores democráticos. Y es que, sin duda, el proyecto de integración económica de los estados se debe desenvolver en virtud de acuerdos esenciales en política económica, pero también, y fundamentalmente, en estricto cumplimiento de los derechos fundamentales de la persona.

\section{DECLARACIÓN DEL MOEA WASHINGTON D.C. Washington D.C. \\ 26 de julio de 2013}

\section{“ESTRATEGIAS PARA MINIMIZAR LA INEQUIDAD EN LOS ESTADOS MIEMBROS, MEDIANTE LA INTEGRA- CIÓN ECONÓMICA REGIONAL DE LAS AMÉRICAS"}

Los Estudiantes de las Américas reunidos en el Trigésimo Primer Modelo de la Asamblea General de la OEA para universidades del Hemisferio (310 MOEA), en la ciudad de Washington D.C., Estados Unidos de América, 
REITERANDO el compromiso asumido por los Estados miembros en la Carta de la OEA, donde se reconoce que uno de los propósitos de la Organización es promover mediante la acción cooperativa el desarrollo económico, social, cultural y tecnológico a través de principios rectores como la solidaridad y la equidad;

RECONOCIENDO el espíritu de la Carta Social de las Américas que promueve la cooperación interamericana y los principios democráticos para luchar contra la corrupción a fin de obtener instituciones sólidas que garanticen la seguridad jurídica;

CONSIDERANDO que la liberación comercial y la inversión privada garantizan el crecimiento económico sostenido;

AFIRMANDO que los Estados miembros tienen la voluntad de establecer una estrategia que combine las políticas para el desarrollo sostenible, con el fin de mejorar de forma equitativa el bienestar y las condiciones de vida de las generaciones presentes y futuras;

CONVENCIDOS de que la educación es un derecho inalienable de los pueblos para promover la generación del capital humano, en el sentido de mejorar sistemas productivos capaces de generar un desarrollo económico;

EXPRESANDO la necesidad de fortalecer las políticas sociales inclusivas desarrolladas por los Estados miembros, en reconocimiento de la diversidad y la promoción de los derechos de los grupos vulnerables del hemisferio,

DECLARAN:

Recomendar a los Estados miembros de la Organización la reducción de las barreras económicas, con la finalidad de facilitar el libre comercio y la integración de los países del hemisferio.

Encomendar a la Secretaría General que coordine e incentive, mediante foros anuales, el diálogo interregional de los sistemas de integración económica del hemisferio.

Recomendar a los países miembros de la Organización impulsar las exportaciones de las micro y pequeñas empresas de las regiones del Hemisferio, implementando sistemas de logística de última generación que conecten el comercio interregional. 
Agregar a la agenda de reuniones ministeriales que se realizan anualmente, el intercambio de información, experiencia y métodos de implementación de prácticas exitosas resultantes de políticas de especialización en los sectores productivos y de infraestructura.

Encomendar a la Secretaría para el Desarrollo Integral que coordine la creación de un programa de intercambio de enseñanza técnica y superior en la región, integrando las experiencias exitosas con el fin de replicar estas experiencias de conocimiento en el Hemisferio.

Solicitar a la Secretaría General que con el apoyo de los Estados miembros realice las acciones necesarias para lograr la implementación de la presente Declaración.

\subsubsection{Comisión General: "Aplicación de los principios de la Carta Social de las Américas para el fortalecimiento de la democracia"}

De acuerdo a la Propuesta de Plan de Acción de la Carta Social de las Américas, las políticas, programas e iniciativas que se desprenden de la Carta Social y su Plan de Acción están inspiradas por los principios de participación, intersectorialidad, solidaridad y cooperación interamericanas, respeto a la diversidad y a los derechos humanos. En ese sentido, el Modelo reforzó la consigna de que la aplicación de estos principios debe orientar siempre el fomento del sistema democrático. Y, en esta ocasión, reconociendo la importancia de un sistema educativo confiable, la Comisión General recordó, a propósito de lo dispuesto en la Carta Democrática Interamericana, que la educación es un servicio clave para fortalecer las instituciones democráticas, promover el desarrollo del potencial humano y el alivio de la pobreza, así como para fomentar un mayor entendimiento entre los pueblos.

Es por ello que instó a lograr una educación de calidad, regida por principios democráticos, que esté al alcance de las mayorías; que, sin perder niveles óptimos de calidad, incluya a las niñas y mujeres, a los habitantes de las zonas rurales y los ciudadanos de las extensas minorías.

\subsubsection{Primera Comisión, Comisión de Asuntos Políticos y Jurídicos: “For- talecimiento de la Carta Democrática Interamericana para garanti- zar procesos electorales libres y justos en los Estados miembros"}

La Carta Democrática Interamericana, adoptada en Lima en 2001, ha sido denominada como un manual de conducta, una guía del compor- 
tamiento democrático que debe ser observado por todos los agentes políticos de la región ${ }^{3}$. En tal sentido, este instrumento se convierte en una de las más importantes herramientas políticas para el respeto de los principios democráticos.

Sobre esa afirmación, atendiendo a la necesidad de promover la justa participación de la población en los sistemas electorales, esta Comisión recogió, en la mayoría de sus resoluciones, el sentido de que la Carta Democrática Interamericana pretende promover, preservar y defender la democracia ante eventuales amenazas.

La Primera Comisión recalcó que el proceso electoral es un microcosmos del sistema democrático, en el cual autoridades y sociedad civil deben velar por el respeto absoluto de los derechos humanos y las libertades fundamentales. Y, con ello, la Comisión cumple con la obligación moral, inherente a todo foro político, de ampliar los alcances prácticos de las instituciones, en este caso, la de considerar que los valores democráticos son también dignos de ser protegidos al amparo de los derechos humanos.

\subsubsection{Segunda Comisión, Comisión de Seguridad Hemisférica: "El Informe sobre Drogas: Identificación de nuevos enfoques para enfrentar el tráfico ilícito de drogas"}

Sin duda, el asunto de las drogas representa uno de los más grandes y difíciles problemas sociales de la región. Como se ha señalado, en tanto fenómeno de comportamiento social, sus relaciones son múltiples, dinámicas y difíciles de precisar (OEA, 2013, p. 5). Es por tal motivo que la identificación de nuevos enfoques para enfrentar el tráfico ilícito de drogas ha resultado ser impostergable.

Las altas tasas de abandono de estudios escolares, la inestabilidad familiar, la inseguridad ciudadana y el serio problema de salud pública fueron motivo de reflexión de los grupos de debate de esta Comisión. Es por ello que las resoluciones fueron, esta vez, instrumentos para la exhortación a la adopción de novedosos mecanismos de lucha contra las dro-

3 La Carta Democrática Interamericana ha sido calificada de esa manera por César Gaviria Trujillo, secretario general de la Organización de los Estados Americanos desde 1994 hasta 2004. Vid. Consejo Permanente de la Organización de los Estados Americanos. Carta Democrática Interamericana: documentos e interpretaciones. Washington D.C.: 2013, p. ix. 
gas. Entre las principales perspectivas se encuentran algunos mecanismos para la reducción de la demanda, los sistemas educativos para la prevención, el fortalecimiento de la seguridad contra el tráfico ilícito y la reestructuración de los ordenamientos penales. Nuevas soluciones que deberían ser trabajadas, como recomienda la Comisión, en un marco apropiado de cooperación entre los estados.

\subsubsection{Tercera Comisión, Comisión de Gestión de Cumbres Interameri-} canas y Participación de la Sociedad Civil en las Actividades de la OEA; y Consejo Interamericano para el Desarrollo Integral (CIDI): "Promoción de la cooperación entre el sector privado y entidades de educación superior y técnica para impulsar el emprendimiento juvenil en las Américas"

Uno de los principales objetivos de esta Comisión fue la reflexión y el debate sobre posibles mecanismos que sirvan para fomentar el empleo e impulsar mercados más competitivos en la región. Así, el trabajo de estos aspectos sociales se llevó a cabo en el marco del respeto de las libertades económicas que rigen la actividad del sector privado. Al respecto, como señala una de las resoluciones aprobadas en esta Comisión, resulta imprescindible para los estados reconocer que "el pensamiento emprendedor permite que los jóvenes conviertan su creatividad, energía e ideas en oportunidades empresariales, ayudándolos a facilitar su entrada en el mercado de trabajo" (MOEA, 2013).

Definitivamente, la naturaleza de la agenda de esta Comisión significó el desarrollo de un interesante análisis acerca de la importancia del sector privado en la sociedad; así como la necesidad de políticas estatales que resalten la estrecha relación entre sistemas educativos justos y posibilidades de acceso a mercados competitivos.

\subsubsection{Cuarta Comisión, Comisión de Asuntos Administrativos y Pre- supuestarios: "Modalidades nuevas y creativas para el financia- miento de la OEA como organización intergubernamental, teniendo en cuenta relaciones con entidades privadas, institucio- nes financieras internacionales, países miembros y observadores de la OEA y otras entidades"}

Con relación a la agenda, esta Comisión consideró preciso tomar en consideración el artículo 11 de la Carta Democrática Interamericana, según el cual se establece que tanto la democracia como el desarrollo 
económico y social son interdependientes y se refuerzan mutuamente. Ello, a fin de promover esfuerzos conjuntos para resolver los problemas financieros pendientes de solución en la OEA.

En ese sentido, ante la necesidad de implementar modelos de financiamiento a través de la colaboración de entidades privadas, para ayudar a superar necesidades financieras, la Comisión recuerda el compromiso moral de los miembros de la OEA. Así, ha resuelto "Invitar a todos los actores involucrados con la Organización de los Estados Americanos, incluyendo a la sociedad civil, el sector privado, las Organizaciones No Gubernamentales y los Observadores Permanentes, a reafirmar el compromiso de cooperación con la Organización" (MOEA, 2013).

\subsection{MOEA}

5.2.1 Declaración de Arequipa: "La educación como elemento primordial para la inclusión social y económica en las Américas"

\section{DECLARACIÓN DE AREQUIPA PARA EL TRIGÉSIMO SEGUNDO MODELO DE LA ASAMBLEA GENERAL DE LA OEA:}

\section{“LA EDUCACIÓN COMO ELEMENTO PRIMORDIAL PARA LA INCLUSIÓN SOCIAL Y ECONÓMICA EN LAS AMÉRICAS"}

LAS Y LOS DELEGADOS ANTE EL 32० MODELO DE LA ASAMBLEA GENERAL DE LA OEA PARA UNIVERSIDADES DEL HEMISFERIO (32० MOEA), reunidos en Arequipa, República del Perú, del 22 al 25 de julio de 2014.

CONSIDERANDO que la Carta de la Organización de los Estados Americanos en sus Artículos 49 y 50, señala la obligación de los Estados de adaptar a sus normas constitucionales las políticas educativas en sus fases primaria $y$ secundaria, a fin de erradicar el analfabetismo y extender la educación a toda la población;

TENIENDO EN CUENTA que la Carta Social de las Américas, en su Capítulo III responsabiliza a los Estados de promover el desarrollo social con igualdad e inclusión, combatiendo toda forma de discriminación, a fin de garantizar la igualdad de oportunidades para todos sus ciudadanos; 
CONVENCIDOS de la importancia central de los y las estudiantes en la educación y enfatizando el rol que asumen los y las docentes en su formación, se reconoce su meritoria y trascendente labor en el progreso y acceso del derecho a una educación digna, así como también en su lucha por la formación de ciudadanos responsables y comprometidos con la sociedad y fomentando en los y las estudiantes aprendizajes significativos, armonía social, diálogo intercultural y respeto a la diversidad, como lo establece la Declaración de Paramaribo, con el fin de superar los logros educativos en beneficio de las y los estudiantes;

CONSCIENTES de que la Declaración del Milenio, suscrita por 189 Jefes de Estado en la Asamblea General de la ONU en el año 2000, incluye dentro de sus objetivos de desarroIlo aspectos vinculados a la erradicación de la pobreza, y teniendo en cuenta entre otros criterios que la Carta Democrática Interamericana en su Capítulo III, Artículo 16 señala que: "la educación es clave para fortalecer las instituciones democráticas, promover el desarrollo del potencial humano y el alivio de la pobreza";

CONSIDERANDO que según el artículo 26 de la Declaración Universal de los Derechos Humanos "la educación es un derecho humano fundamental", y en concordancia con la Carta de la OEA que en su Artículo 47 declara que: "Ios Estados miembros darán importancia primordial, dentro de sus planes de desarrollo, al estímulo de la educación, la ciencia, la tecnología y la cultura como fundamento integral de la persona, de la democracia, la justicia social y el progreso";

DESTACANDO que la cooperación interamericana para el desarrollo integral es responsabilidad común y solidaria de los Estados miembros, en el marco de los principios democráticos, y que la Organización de los Estados Americanos a través de su Consejo Interamericano para el Desarrollo Integral tiene como finalidad promover la cooperación entre los Estados miembros con el propósito de lograr su desarrollo integral y, en particular, contribuir a la eliminación de la pobreza;

FUNDAMENTANDO que la cooperación internacional encuentra su razón de ser en los principios universales de solidaridad entre los pueblos, respeto y protección de los derechos humanos, y en la búsqueda incesante de mejores condiciones y mayores recursos que brinden al ser humano 
una situación de bienestar y seguridad conforme a su dignidad, siendo éste el fin último de la existencia de los Estados.

DECLARAN:

ALENTAR Y FORTALECER las políticas públicas de inclusión social que mejoren el acceso a la educación de calidad justa y equitativa, aumentando progresivamente la cobertura territorial con la finalidad de eliminar la brecha urbano-rural, bajo ningún tipo de discriminación, con énfasis en la no discriminación de género, y manteniendo la identidad cultural de los Estados.

REAFIRMAR que una inversión adecuada en educación constituye una prioridad para el desarrollo armónico e integral de nuestros pueblos, teniendo en cuenta que la cooperación interestatal con organismos internacionales y mecanismos de integración constituye una herramienta adecuada para el logro de dicho fin.

MEJORAR la calidad de la educación que proveen los Estados miembros principalmente en los grupos vulnerables de cada país a través de una formación continua; una constante actualización mediante el uso de las TICs y la capacitación de docentes; poniendo énfasis en las estrategias pedagógicas así como en la movilidad de docentes mediante una mayor publicidad de becas existentes brindadas por la OEA, otros organismos internacionales y agencias de cooperación, para docentes que enseñen en los niveles básico, medio, técnico y superior, de manera que no exista una desvinculación con la realidad en el proceso educativo. Asimismo, promover el intercambio de docentes en el Hemisferio para integrar el conocimiento adquirido en el país de origen y en el país de destino, para que puedan llevar a las y los estudiantes una visión más amplia del conocimiento que se desea transmitir.

COMPENSAR según las posibilidades de cada Estado la dedicación y esfuerzo brindados por las y los docentes, mediante una digna remuneración. Del mismo modo, realizar planes nacionales de incentivos salariales para docentes de las escuelas públicas y privadas ubicadas en zonas de difícil acceso; otorgando reconocimientos a los docentes que hayan destacado en su labor, con el objetivo de promover la excelencia del sistema educativo.

FORTALECER el compromiso de los Estados miembros de la OEA para que por medio de la erradicación de la pobreza, 
según el contexto socioeconómico de cada país, se logre que la población tenga mayor acceso a la educación.

REDOBLAR los esfuerzos de los Estados miembros para:

a) Mejorar la calidad y cobertura de los programas de nutrición estudiantil en niñas y niños;

b) Garantizar el acceso y permanencia en toda la etapa escolar con el propósito de que más personas terminen su formación escolar, y

c) Fomentar la capacitación técnica en las y los adolescentes, así como programas de alfabetización adulta ya existentes en los Estados miembros, y considerar la creación de dichos programas en aquellos países en los que no existieran, atendiendo a sus posibilidades.

ENCOMENDAR a la OEA, a través de la Comisión Interamericana de Educación ( $\mathrm{CIE}$ ), con apoyo de su Secretaría Técnica, analizar la situación de cada uno de los países que lo soliciten y sugerir la creación y/o fortalecimiento de los programas anteriormente mencionados, buscando su sostenibilidad y financiamiento mediante proyectos de cooperación regional y/o internacional. Por último, encomendar a la CIE a través de su Secretaría Técnica, llevar a cabo consultas a los Estados miembros sobre los proyectos mencionados para replicar las buenas prácticas.

\subsubsection{Temario}

En el XXXII MOEA, el temario que se estableció fue el siguiente:

a) Comisión General: "Establecimiento de mecanismos que fomenten la participación y el liderazgo político de la mujer en el Hemisferio".

b) Primera Comisión: “Gobernabilidad democrática a través del fortalecimiento de los gobiernos locales en los Estados miembros".

c) Segunda Comisión: "Desarrollo de estrategias de prevención, respuesta y gestión de crisis ante amenazas bioterroristas en las Américas".

d) Tercera Comisión y CIDI: “Gestión integrada de recursos hídricos como elemento promotor del desarrollo en las Américas".

e) Cuarta Comisión: "Mecanismos para disminuir la brecha entre los ingresos y egresos de la OEA para cubrir gastos de contingencia de la Organización y proyectos de prioridad en el Hemisferio". 


\section{Conclusiones}

Desde su creación, el MOEA viene siendo uno de los principales espacios académicos de nuestro continente diseñado para estudiantes universitarios preocupados por los asuntos políticos más importantes de la región. Y, en esta oportunidad, los edificios de la Organización en Washington D.C. y el campus de la Universidad Católica San Pablo en Arequipa, se convirtieron en lugares de reflexión, crítica y sano debate sobre democracia, pobreza, educación, derechos humanos, corrupción, inclusión social, entre otros temas.

Sin duda, durante los cuatro días en los que se llevó a cabo el Modelo, muchos de los estudiantes vimos satisfecho el deseo de asumir un rol protagónico en la solución de los problemas más serios de la región. Así, al mismo tiempo que fortalecíamos nuestras aptitudes académicas e interpersonales, inventábamos con seriedad y compromiso el continente que queremos sea América.

De esta manera, si bien fuimos en todo momento conscientes de que el derecho internacional no reviste formas de coacción tradicionales, regresamos a nuestras ciudades con la confianza de que todas aquellas recomendaciones plasmadas tanto en las declaraciones como en las resoluciones aprobadas, tienen en el respeto de la moral y la dignidad del ser humano las más nobles garantías.

\section{Referencias}

González, D. [12 de mayo 2012]. Mi visita a la OEA, Washington, D.C. y ¿qué es el MOEA? [en línea]. <http://www.youtube.com/watch?$\mathrm{v}=x a y f H 0 \mathrm{~ms} 3 \mathrm{Ss}>$. [Consulta: 17 de enero de 2014].

MOEA. (2013). La inserción de la cultura emprendedora en el plan de estudio de la región. AG/Res. III-6

MOEA. (2013). "Programa participativo para la obtención y optimización de los recursos de la Organización de los Estados Americanos". AG/Res. IV-1

Organización de los Estados Americanos. (S.f.). Asamblea General [en línea]. Acerca de la OEA. Nuestra Estructura. < http:// www.oas.org/es/acerca/asamblea_general.asp>. [Consulta: 20 de enero de 2014]. 
Organización de los Estados Americanos. (S.f.). Carta Democrática Interamericana [en línea]. Capítulo III: “Democracia, desarrollo integral y combate a la pobreza". <http://www.oas.org/charter/ docs_es/resolucion1_es.htm>. [Consulta: 20 de enero de 2014].

Organización de los Estados Americanos. (S.f.). Comisión General [en línea]. Consejo Permanente de la Organización de los Estados Americanos. <http://www.oas.org/consejo/sp/COMGRAL/ default.asp>. [Consulta: 20 de enero de 2014].

Organización de los Estados Americanos. (S.f.). Comisión de Asuntos Jurídicos y Políticos [en línea]. Consejo Permanente de la Organización de los Estados Americanos. <http://www.oas.org/consejo/ sp/CAJP/default.asp >. [Consulta: 20 de enero de 2014].

Organización de los Estados Americanos. (S.f.). Comisión de Seguridad Hemisférica [en línea]. Consejo Permanente de la Organización de los Estados Americanos. <http://www.oas.org/csh/spanish/ default.asp>. [Consulta: 20 de enero de 2014].

Organización de los Estados Americanos. (S.f.). Modelo de la Asamblea General de la OEA (MOEA) [en línea]. < http://www.oas.org/es/ sga/moea/default.asp $>$. [Consulta: 20 de enero de 2014].

Organización de los Estados Americanos. (S.f.). Nuestra historia [en línea]. Acerca de la OEA. <http://www.oas.org/es/acerca/nuestra_historia.asp>. [Consulta: 20 de enero de 2014].

Organización de los Estados Americanos. (S.f.). Quiénes somos [en línea]. Acerca de la OEA. <http://www.oas.org/es/acerca/quienes_somos.asp >. [Consulta: 20 de enero de 2014].

Organización de los Estados Americanos. (2013). El problema de drogas en las Américas: Estudios. Drogas y seguridad. Washington, D.C. 